\title{
The perceived impact of publications on Neglected Tropical Zoonoses as measured by their impact factor
}

\author{
Dieter Vanderelst • Sara Speybroeck • Niko Speybroeck
}

Received: 15 December 2010/Published online: 9 September 2011

(C) Akadémiai Kiadó, Budapest, Hungary 2011

\begin{abstract}
We investigated whether papers on Neglected Tropical Zoonoses are published in journals with lower impact factors than research on diseases with a similar global health burden. We found that, despite being cited equally often, the papers on Neglected Tropical Zoonoses were published in journals with lower impact factors. The scopes of these journals are mainly restricted to Tropical medicine. A clustering analysis revealed that The Lancet, a high impact general medical journal, does pay attention to Neglected Tropical Zoonoses. We discuss our findings in the context of the ongoing discussion about the publishing policies of medical journals. Moreover, our findings stress the importance of recent suggestions that impact factors should not be used for assigning public funding to research (programs) on Neglected Tropical Zoonoses.
\end{abstract}

Keywords Neglected Tropical Zoonoses - Neglected Tropical Diseases · DALY · Burden of disease

\section{Introduction}

According to the WHO, zoonoses are diseases and infections that are naturally transmitted between vertebrate animals and humans. A subgroup of these diseases have been termed Neglected Tropical Zoonoses (e.g. Hotez et al. 2007b). Indeed, except for the newly emerging zoonoses such as SARS and highly pathogenic avian influenza H5N1, the vast majority of zoonoses affect mostly the poorest populations and are not prioritized by health

D. Vanderelst $(\bowtie)$

Department of Applied Economics, University Antwerp, Antwerp, Belgium

e-mail: dieter.vanderelst@ua.ac.be

S. Speybroeck

Instituut voor Onderwijskunde \& Informatie Wetenschappen, University Antwerp, Antwerp, Belgium e-mail: Sara.Speybroeck@ua.ac.be

N. Speybroeck

Faculté de Santé Publique, Université Catholique de Louvain, Louvain, Belgium

e-mail: Niko.Speybroeck@uclouvain.be 
systems at national and international levels (Hotez et al. 2009; WHO 2011). This group of diseases include conditions such as bovine tuberculosis, brucellosis and cysticercosis (WHO 2011).

The neglect of these diseases is reflected by the fact that their impact on global health is greatly underestimated (Mathers et al. 2007; Engels and Savioli 2006; Hotez et al. 2007a, b), mostly due to a lack of data (Mathers et al. 2007; Engels and Savioli 2006). Furthermore, it has been argued that virtually no new drugs are being developed for this group of conditions (Yamey 2002; Chirac and Torreele 2006; Médecins Sans Frontières 2001). Finally, news coverage of these diseases is sparse (Balasegaram et al. 2008) and public attention low (Vanderelst and Speybroeck 2010).

Recently, we found that the lack in attention for Neglected Tropical Zoonoses is also reflected in scientometric indicators. We have shown that far less papers are being published on a number of Neglected Diseases than on non-neglected diseases with a similar global health impact (Vanderelst and Speybroeck 2010). Both the neglected and nonneglected diseases included in this previous study had a similar global health impact. However, the impact of the Neglected Diseases was not evenly distributed across the world population. The impact of the neglected diseases was much higher among poor populations. Therefore, our 2010 data showed that scientific effort is skewed towards diseases affecting more privileged populations.

In this paper, we extend our research into the scientometrics of Neglected Tropical Zoonoses by comparing the impact factors and number of citations of papers on Neglected Tropical Zoonoses and conditions with a matched global health impact. This is, we compare the impact factors for publications on Neglected Tropical Zoonoses with those of publications on a number of conditions that worldwide have the same impact on the disability and mortality of people.

The reason for this analysis depends on an assumption about the significance of the impact factor. In this study, the impact factor is assumed to be a proxy for the perceived importance of a research topic. The impact factor is a journal level metric bearing little information about the impact of individual papers (Frank 2003; Seglen 1997; Pringle 2008). Nevertheless, researchers in medicine (and other fields) are increasingly evaluated based on the impact factors of the journals they publish in (see Seglen 1997, for an overview). The reason for this is that scientific journals with a higher impact factor are perceived as more prestigious and harder to publish in (e.g. Saha et al. 2003; Catling et al. 2009; Regazzi and Aytac 2008). Moreover, editors of journals are known to select those publications that are likely to increase the impact factor of the journal by being cited often (Krell 2010; Chew et al. 2007; Matias-Guiu and Garcia-Ramos 2008). Finally, funding agencies often use impact factors when judging the importance of scientific output (see Morel et al. 2009, for a discussion in the context of public health).

Therefore, the impact factor of the journals in which research is published can be used as a proxy for the importance of research as judged by editors, researchers and funding agencies.

Besides the impact factor of papers, we also analysed the number of citations to papers on Neglected Tropical Zoonoses and on a number of conditions with the same global health impact. In the context of this paper, we consider the number of citations as an indicator of the realised impact of a paper on scientific progress. In contrast to the impact factor, which is a measure aggregated across all papers published in a journal, the number of citations is a direct measure of the performance of a paper. We included an analysis of the number of citations to check whether any discrepancies in the impact factors between 
the two classes of papers could be attributed to differences in quality and scientific impact between the two classes of papers.

\section{Methods}

Since 1990, the World Health Organization uses the disability-adjusted life year (DALY) statistic to quantify the burden of diseases and to determine priorities for interventions (Mathers et al. 2007). The DALY attempts to quantify the impact of a disease on global health (Pruss-Ustun et al. 2003), taking into account both global mortality and disability due to a disease. Currently, the DALY is the most comprehensive statistic by which global health can be quantified.

We selected 11 Neglected Tropical Zoonoses and 11 conditions with a matched DALY (WHO 2008). The DALYs for both groups of diseases did not differ significantly according to a Paired Exact Wilcoxon signed rank test $(V=50, P=0.15)$. The selected conditions and their DALY estimates are listed in Table 1. More details about the selection of the diseases are reported by Vanderelst and Speybroeck (2010).

It should be noted that, while both groups of diseases have a similar global health impact, they differ in the populations they affect. Figure 1, plots the average DALY per person for the two groups of diseases. From this graph, it can be seen that the Neglected Tropical Zoonoses mainly affect people with the lowest income (Kruskal-Wallis rank sum test $\left.\left[\chi^{2}(3)=23, P<0.01\right]\right)$. In contrast, the impact of the matched diseases is more equally distributed across income groups (Kruskal-Wallis rank sum test $\left[\chi^{2}(3)=0.53\right.$,

Table 1 The 11 selected Neglected Tropical Zoonoses and matched conditions used in this paper together with their 2004 DALY estimate (WHO 2008)

\begin{tabular}{|c|c|c|c|c|c|c|c|c|}
\hline $\begin{array}{l}\text { Neglected } \\
\text { Zoonoses }\end{array}$ & DALY & $\begin{array}{l}\text { Hotez } \\
(2007 b)\end{array}$ & $\begin{array}{l}\mathrm{DP} \\
\mathrm{P}_{\mathrm{L}}\end{array}$ & $\begin{array}{l}\mathrm{DP} \\
\mathrm{P}_{\mathrm{H}}\end{array}$ & Matched diseases & DALY & $\begin{array}{l}D P \\
P_{L}\end{array}$ & $\begin{array}{l}\mathrm{DP} \\
\mathrm{P}_{\mathrm{H}}\end{array}$ \\
\hline Leprosy & 194 & 200 & 0.066 & 0.000 & Poliomyelitis & 34 & 0.011 & 0.004 \\
\hline Onchocerciasis & 389 & 500 & 0.159 & 0.000 & Diphtheria & 174 & 0.067 & 0.000 \\
\hline Chagas disease & 430 & 700 & 0.001 & 0.002 & $\begin{array}{c}\text { Periodontal } \\
\text { disease }\end{array}$ & 320 & 0.067 & 0.039 \\
\hline Dengue & 670 & N/A & 0.165 & 0.006 & Appendicitis & 418 & 0.080 & 0.036 \\
\hline $\begin{array}{l}\text { Japanese } \\
\text { encephalitis }\end{array}$ & 681 & N/A & 0.199 & 0.003 & $\begin{array}{l}\text { Vitamin A } \\
\text { deficiency }\end{array}$ & 629 & 0.243 & 0.000 \\
\hline Trichuriasis & 1012 & 6400 & 0.274 & 0.006 & Hepatitis C & 955 & 0.197 & 0.157 \\
\hline $\begin{array}{l}\text { Hookworm } \\
\text { disease }\end{array}$ & 1092 & 22100 & 0.239 & 0.012 & Bladder cancer & 1451 & 0.125 & 0.446 \\
\hline Trypanosomiasis & 1673 & 1500 & 0.657 & 0.000 & Multiple sclerosis & 1527 & 0.198 & 0.327 \\
\hline Schistosomiasis & 1707 & 4500 & 0.619 & 0.009 & Parkinson disease & 1710 & 0.143 & 0.711 \\
\hline Ascariasis & 1851 & 10500 & 0.590 & 0.006 & Ovary cancer & 1745 & 0.207 & 0.473 \\
\hline Leishmaniasis & 1974 & 2100 & 0.771 & 0.004 & Hepatitis B & 2068 & 0.512 & 0.084 \\
\hline
\end{tabular}

The DALYs have been scaled by a factor $1 / 1,000$. The columns labelled DP $\mathrm{P}_{\mathrm{L}}$ and $\mathrm{DP} \mathrm{P}_{\mathrm{H}}$ the fifth column give the worldwide DALY per capita for the lowest and the highest income groups as defined by the World Bank. The column labelled Hotez (2007b) lists the updated DALY estimates for the Neglected Tropical Zoonoses as given by Hotez et al. (2007b). Note that, for some diseases, these figures are considerably higher than the WHO estimates 
Fig. 1 The average DALY per person for the two groups of diseases included in this study as a function of income level. The four groups contain countries with a low (US\$825 or less), lower middle (US\$ 826-3.255), upper middle (US\$

3.256-10.065) or high (US\$ 10.066 or more) gross national income (GNI) per capita respectively. These income categories are defined in the the World Bank's World Development Report 2004

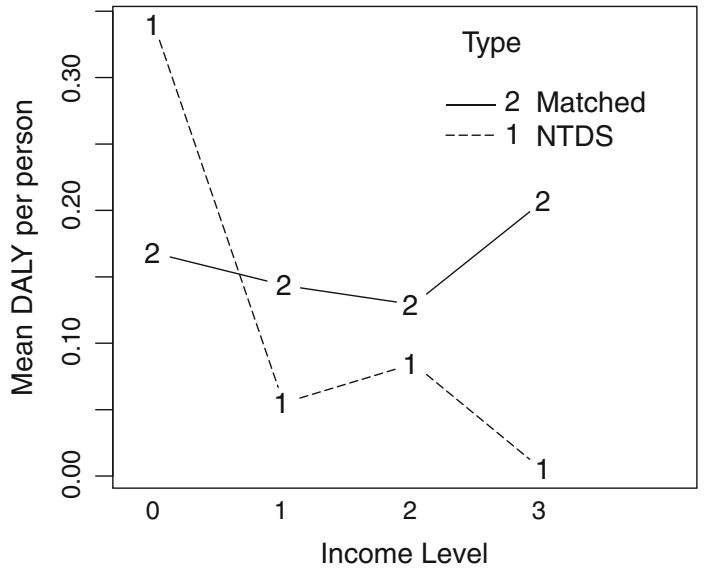

$P=0.91])$. Indeed, these diseases tend to affect the most wealthy populations slightly more.

We queried the PubMed database for journal papers relating to the Neglected Tropical Zoonoses and the matched conditions published between January 1998 and December 2009. For each paper we retrieved the journal International Standard Serial Number field. In particular we used the linking ISSN or ISSN-L as this is the same for different media versions (electronic vs. paper) of a journal (US National Library of Medicine 2010) and is used by the ISI Web of Science.

From the ISI Web of Science, we retrieved the Impact Factors as listed in the Journal Citation Reports ${ }^{1}$ for all journals listed in the science index together with their ISSN numbers. Where available, we retrieved the Impact Factors from 1998 to 2009. This is the complete range of available Impact Factors at the time this research was carried out. We attempted to assign to each paper retrieved from PubMed the Impact Factor of the journal in which it was published at its year of publication by matching the ISSN numbers. This was not possible for each paper as PubMed also lists journals that are not listed in ISI Web of Science (Falagas et al. 2008). Papers published in a journal that was not listed by the Web of Science in the year of their publication were assigned an impact factor of 0.024. This was the lowest impact for any journal in our dataset. Imputing the data in this way reflects the assumption that journals that are not listed in the Web of Science have a very low impact factor and allowed us to take into account publications that were not listed in ISI Web of Science. It should be noted that analysing the data without imputation yielded similar results as those reported here.

Finally, we queried the Web of Science for the number of citations to the papers published on the 22 diseases included in the current study. We retrieved the number of citations to the papers published between 1998 and 1990 up to May 2010 using the citation analysis tool provided on the website.

Both Impact Factors and number of citations are skewed. To be able to analyse the data using parametric methods, the Impact Factors and number of citations were transformed using the maximum likelihood Box-Cox transformation (Venables and Ripley 2002). This led to more normally distributed data. All statistical analyses were performed on the

1 Thomson Reuters Journal Citation Reports: http://thomsonreuters.com/products_services/science/science_ products/a-z/journal_citation_reports/ 
transformed data. Non-Parametrical tests were also performed where possible and these confirmed the results reported below.

To investigate in more detail whether research on the two classes of diseases is published in different journals, we performed two hierarchical clustering analyses. We build a contingency table listing the number of articles that appeared on each of the diseases in each of the journals in our dataset. From this contingency table we selected the 10 most published in journals for both classes of conditions. From the selected data, two distance matrices were build listing the Euclidean distances between the journals and the diseases. The distance between two journals is small when they tend to publish papers on the same diseases. Similarly, the distance between two diseases is small if research on them tends to be published in the same journals. Next, we used a hierarchical clustering algorithm to visualize both distance matrices.

\section{Results}

Missing data

Not all papers retrieved in PubMed could be assigned a Web of Science impact score (see Fig. 3a). The proportion of papers for which no impact factor could be retrieved did not vary across years as tested using the Kruskal-Wallis rank sum test $\left[\chi^{2}(11)=11\right.$, $P=0.44]$. Also, we could not find a statistical significant difference between the two classes of diseases in the proportions of missing papers. For each year, a Kruskal-Wallis rank sum test yielded a non-significant result $\left[\chi^{2}(1)<1.5, P>0.05\right]$.

\section{Cluster analysis}

Table 2 lists the ten most frequent journals for the two classes of diseases. This table shows that papers on Neglected Tropical Zoonoses are most often published in journals focusing on tropical medicine. Only two of the most frequently published in journals are not restricted to tropical medicine: Parasitology Research and Parasitology.

Papers on the matched conditions are published in topical journals that do not focus on a geographical region but on a sub-discipline of medicine (e.g. oncology). Furthermore, one of the most frequent journals for the matched diseases is a general medical journal (The Lancet).

In a cluster analysis, we investigated the difference between the journals in which research on both classes of diseases is published in more detail. The clustering algorithm used was the hclust method as provided by the statistical package $\mathrm{R}^{2}$ (R Development Core Team 2010) using complete linkage. This analysis (Fig. 2) confirms that papers on Neglected Tropical Zoonoses tend to be published in different journals than those on the matched conditions. Only papers on Dengue and Japanese Encephalitis are published in similar journals as the research on the matched diseases. Similarly, the ten most used journals for both classes of diseases clustered in a specific way. Journals publishing on Neglected Tropical Zoonoses clustered together while the distances between the journals publishing on the matched diseases were larger. Only the fairly general journals The Lancet and Vaccine had a publication profile more similar to the journals publishing mostly on Neglected Tropical Zoonoses. The cluster analysis revealed that the two journals that do

$\overline{{ }^{2} \text { R-project: http://www.r-project.org/ }}$ 
Table 2 The ten most frequently published in journals for each category of diseases (top: Neglected Tropical Zoonoses, bottom: matched diseases

\begin{tabular}{|c|c|c|c|}
\hline ISSN & Percentage & $\begin{array}{l}\text { Impact } \\
\text { factor }\end{array}$ & Title \\
\hline $0002-9637$ & 3.02 & 2.907 & The American Journal of Tropical Medicine and Hygiene \\
\hline $0035-9203$ & 2.84 & 2.518 & Transactions of the Royal Society of Tropical Medicine and Hygiene \\
\hline 0074-0276 & 2.09 & 1.858 & Memorias do Instituto Oswaldo Cruz \\
\hline $0001-706 X$ & 2.04 & 2.446 & Acta Tropica \\
\hline 0003-4983 & 1.93 & 1.590 & Annals of Tropical Medicine and Parasitology \\
\hline $0031-1820$ & 1.83 & 2.471 & Parasitology \\
\hline $0125-1562$ & 1.83 & $\mathrm{NA}^{\mathrm{a}}$ & The Southeast Asian Journal of Tropical Medicine and Public Health \\
\hline $1360-2276$ & 1.75 & 2.798 & Tropical Medicine and International Health \\
\hline $0037-8682$ & 1.39 & $0.736^{\mathrm{b}}$ & Revista da Sociedade Brasileira de Medicina Tropical \\
\hline $0932-0113$ & 1.34 & 1.679 & Parasitology Research \\
\hline $0264-410 X$ & 1.49 & 3.508 & Vaccine \\
\hline $0028-3878$ & 0.89 & 7.199 & Neurology \\
\hline $0022-3492$ & 0.88 & 2.488 & Journal of Periodontology \\
\hline 0885-3185 & 0.75 & 3.887 & Movement Disorders \\
\hline $0090-8258$ & 0.75 & 3.402 & Gynecologic Oncology \\
\hline $0022-3166$ & 0.63 & 4.543 & The Journal of Nutrition \\
\hline $0022-5347$ & 0.63 & 4.021 & The Journal of Urology \\
\hline $0140-6736$ & 0.62 & 29.443 & The Lancet \\
\hline 0303-6979 & 0.61 & 3.197 & Journal of Clinical Periodontology \\
\hline $1352-4585$ & 0.58 & 3.280 & Multiple Sclerosis \\
\hline
\end{tabular}

The percentage in column two gives the percentage of articles in the dataset that were published in each of the listed journals. The impact factor is the 5-year impact factor in 2009 as given by the Web of Science

a This journal is not indexed by the web of science

b This journal was only indexed since 2008. The 2009 impact factor is given

not focus explicitly on tropical medicine (Parasitology Research and Parasitology) have a publication profile that is very similar to the journals that do focus on tropical medicine. The two journals ended up in the cluster containing these journals.

Impact factor and citations

Being published in a different set of journals, the impact factors (see Fig. 3b; Table 3) of the papers on matched conditions were higher than those on the Neglected Tropical Zoonoses. Additionally, the impact factors of the papers on both classes of diseases rose over the years. No significant interaction effect between the class disease and year was found indicating a similar trend across time in both classes.

The number of times papers were cited (see Fig. 3c; Table 4) per year did not differ between the Neglected Tropical Zoonoses and the matched conditions. The average number of citations per year depended on the publication year for both classes of diseases. No significant interaction effect between type of disease and publication year could be found.

To evaluate whether the differences in median impact scores between the two classes of diseases represent a meaningful difference, we converted them to percentiles. We extracted 



Fig. 2 (color online) Left dendrogram visualizing the (dis)similarity of the diseases as to in which journal research on them is published. Purple diseases are Neglected Tropical Zoonoses. Orange conditions are matched diseases. Right dendrogram visualizing the (dis)similarity of the journals as to on which diseases they publish articles. Only the 20 journals listed in Table 1 are included in this clustering. Titles printed in purple are journals in which most of the research on Neglected Tropical Zoonoses was published. Orange journals are journals that published many papers on the matched conditions

the distribution of the impact factors for all ISI listed science journals for each year. This distribution was used to estimate the percentile score for the median impact factors plotted in Fig. 3b. It was found that the differences in median impact scores amounted to a differences of about 10 percentiles for each year (see Fig. 3d). For example, while the median impact factor for the papers on Neglected Tropical Zoonoses was higher than about $72 \%$ of the impact factors of all journals the median impact factor for the matched diseases was higher than about $83 \%$ of the impact factors of the same journals. This analysis reveals that, while the differences in median impact factor between the two classes of diseases is small in absolute terms, they represent a detectable shift when considered against the distribution of impact factors.

\section{Discussion}

Of the worlds poorest 2.7 billion people (defined as those who live on less than US\$2.00 a day), more than 1 billion are affected by one or more neglected tropical disease. These diseases not only survive and spread in conditions of poverty, they also exacerbate and perpetuate the poverty of affected communities (WHO 2010). Recently, we have found that, in contrast to the considerable burden represented by these diseases, one of the factors reflecting the neglect of these diseases is the attention paid by science to them. In this paper, we further evaluated the treatment of these conditions by the scientific community by investigating whether the perceived scientific impact of papers on Neglected Tropical Zoonoses is lower than for a set of diseases with a similar global health burden. As outlined 

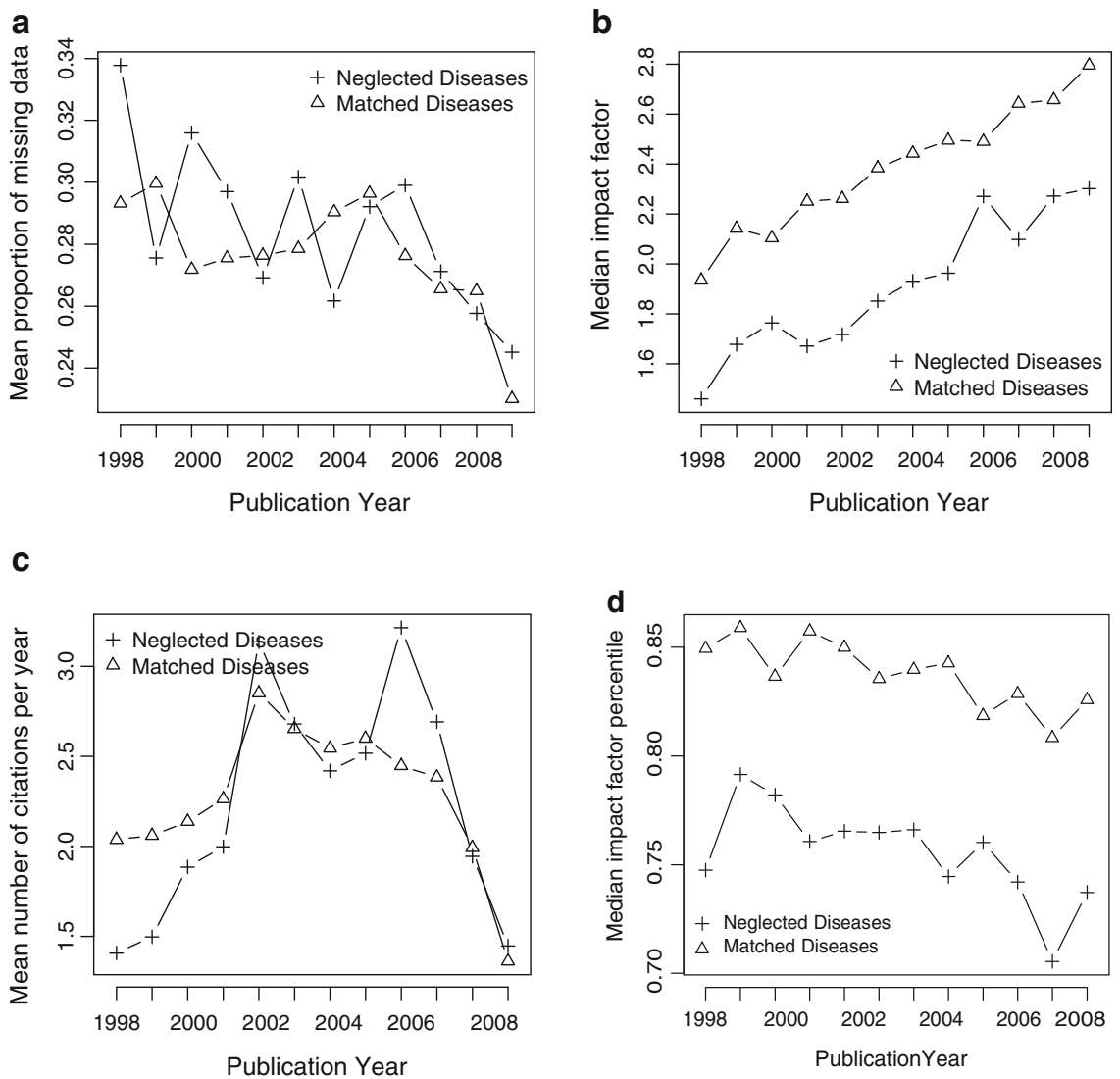

Fig. 3 a Scatterplot of the average proportion of papers to which no ISI impact factor could be assigned as a function of year and the class of disease. $\mathbf{b}$ Scatterplot of the mean impact factor of the papers as a function of the publication year and the class of disease. $\mathbf{c}$ The average number of citations per year to the papers as a function of publication year and the class of disease. d Median percentile of the impact factors of both classes of papers in the distribution of all ISI listed science journals of the corresponding year

Table 3 Results of the Repeated Measures ANOVA performed on the transformed impact factor data

\begin{tabular}{lrrrr}
\hline & Df & Mean square & \multicolumn{1}{l}{$F$} & $P$ \\
\hline Residuals & 10.00 & & 4.25 & \\
Class & 1.00 & 11.39 & 22.31 & 0.00 \\
Year & 11.00 & 3.07 & 6.02 & 0.00 \\
Class:year & 11.00 & 0.43 & 0.84 & 0.60 \\
Residuals & 230.00 & 0.51 & & \\
\hline
\end{tabular}

in the introduction, we assume that the impact factor can serve as a proxy for perceived importance (Regazzi and Aytac 2008; Catling et al. 2009).

Our data confirm that papers on Neglected Tropical Zoonoses are published in a different set of journals than research on diseases with a comparable impact on global health. Out of the ten journals that published most often on the research on Neglected Tropical 
Table 4 Results of the Repeated Measures ANOVA performed on the transformed citation data

\begin{tabular}{lrlll}
\hline & \multicolumn{1}{l}{ Df } & Mean square & $F$ & $P$ \\
\hline Residuals & 10.00 & 6.33 & & \\
Class & 1.00 & 0.95 & 1.32 & 0.25 \\
Year & 11.00 & 4.44 & 6.18 & 0.00 \\
Class:year & 11.00 & 0.57 & 0.79 & 0.65 \\
Residuals & 230.00 & 0.72 & & \\
\hline
\end{tabular}

Zoonoses, eight are journals whose scope is explicitly limited to tropical medicine (Table 1, exceptions: Parasitology and Parasitology Research).

Performing cluster analyses revealed some additional facts about the publication policies of journals. The Lancet and Vaccine are two of the journals that publish most often on the matched diseases. Nevertheless, our cluster analysis suggests that these journals have an overall publication profile that resembles that of the journals publishing on tropical diseases. This indicates that The Lancet and Vaccine, both established general medical journals, pay ample attention to diseases affecting "the bottom billion" (Hotez et al. 2009) (see also Sumathipala et al. (2004) for a similar finding regarding The Lancet).

The cluster analysis revealed differences in the publication profiles among the group of Neglected Tropical Zoonoses. Our results indicate that research on the 11 Neglected Tropical Zoonoses is published in the same group of journals except for papers on Dengue and Japanese encephalitis (Fig. 2). Both diseases are endemic in large regions of the world and not only in Sub-Saharan Africa. According to the World Health Organization, Dengue occurs in more than 100 tropical and sub-tropical countries around the world. Similarly, Japanese encephalitis occurs throughout Asia. The fact that these two diseases have a wide geographical distribution might explain the attention paid to them by journals normally publishing on the matched conditions. Indeed, their estimated global burden is actually much lower than for other Neglected Tropical Zoonoses such as trypanosomiasis (see Table 1) and can not explain the higher amount of attention paid to these conditions.

In short, the clustering analyses showed that (1) at least some general medical journals do pay attention to Neglected Tropical Zoonoses and (2) that not all Neglected Tropical Zoonoses are being ignored by medical journals to the same degree-the geographical distribution of the particular disease might enhance its perceived importance even when its global health impact is lower than other diseases.

It might be argued that it is normal that papers on Tropical Diseases are published more in journals of Tropical Medicine, just as papers on any other specific disease be published more in journals related to that condition. However, higher impact general journals focussing on infectious diseases exist. Indeed, the research could have been published in more general journals such as Trends in Parasitology, Emerging Infectious Diseases and Virology (none of these journals feature in Table 2). While these high impact journals do exist, they are not the most published in journals. Apparently, these journals publish mostly on non-tropical Infectious Diseases. Therefore, topical high impact journals exist but they do not publish on Tropical Medicine.

The fact that research on the two classes of diseases is published in different journals results in a lower impact factor for articles on Neglected Tropical Zoonoses (Fig. 3b). Under the assumptions explained in the introduction, this indicates that the perceived scientific impact of papers on Neglected Tropical Zoonoses is lower. In contrast, the papers on Neglected Tropical Zoonoses and the matched diseases were found to be cited equally often. Taking the number of citations as a measure of realized scientific impact, our data 
indicates that the impact of both classes of papers is the same. In summary, there is a discrepancy between the impact of the papers as measured by the impact factor and by the number of citations.

The presented data does not allow us to infer the mechanism behind the lower impact factors of papers on Neglected Tropical Zoonoses. It is unclear whether this is caused by the submission behavior of authors or the selections made by editors. Evidence suggests that both mechanisms are plausible. Older studies suggest that authors do not necessarily submit to the journals with the highest impact factors. They also take into account other factors such as the readership of a journal (Gordon 1984; Luukkonen 1992). However, the increased availability of search engines might render the selection of a journal less dependent on it readership. More recent studies have found that editors of medical journals actively seek out and select the research that is both of interest to their readers and likely to increase the impact of the journal (Chew et al. 2007; Krell 2010). Given that editors of leading medical journals are almost exclusively recruited among high income groups, they might be biased towards selecting research on health priorities of the rich world (Horton 2003; Bakker and Rigter 1985). Indeed, in a sharp editorial Horton (2003) has warned against the institutional racism exhibited by major medical journals.

Irrespective of the mechanism leading to lower impact factors for research on Neglected Tropical Zoonoses, the question arises whether this has any consequences for the funding and planning of basic research. Lower impact factors might negatively influence the ability for researchers in the field of Neglected Tropical Zoonoses to secure funding by public funding agencies (Hunter 2009). This is especially worrying given the precarious funding situation of Neglected Tropical Zoonoses. Indeed, private investments into Neglected Tropical Zoonoses are very low and insufficient (Yamey 2002; Chirac and Torreele 2006; Médecins Sans Frontières 2001; Bosman and Mwinga 2000). Therefore, consequences would be grave if funding would be even more hampered because of misusing the Impact Factors in judging the importance of basic research into cures, prevention and epidemiology of Neglected Tropical Zoonoses. Alternatives to using the Impact Factor for research evaluations exist and they have been applied for the allocation of funding for research on Neglected Tropical Diseases (Morel et al. 2009). Our data highlights the importance of such endeavours.

While currently our data is insufficient to conclude that the lower impact factors have an effect on the funding decisions of public bodies, our data warrants further investigation of the scores of Neglected Tropical Zoonoses research on various bibliometric indicators. If additional bibliometric markers would exhibit a similar pattern, the risk of reduced funding increases, especially in the light of lacking national and international priories being set (WHO 2011). Moreover, our data contributes to the current debate about the treatment of diseases of poverty by medical journals and the research community (see Horton 2003 for an overview and references).

Finally, it should be noted that the data presented here should be considered as a lower estimate of the impact factor gap between Neglected Tropical Zoonoses and non Neglected Diseases.

First, the DALYs used to match the Neglected Tropical Zoonoses to the other conditions should be considered to be lower estimates. Updated estimations of the DALYs for certain Neglected Tropical Zoonoses have turned out to be much higher (see Table 1).

Second, in this paper, we have only evaluated citations as recorded in the ISI web of science. Previously, it has been established that the number of citations to papers on Neglected Tropical Zoonoses is being underestimated more by the ISI Web of Science for papers on Neglected Tropical Zoonoses than on the matched conditions (Hunter 2009). 
Therefore, the number of citations to the papers on Neglected Tropical Zoonoses might actually be higher for the matched conditions. In this paper we have used the impact factors and number of citations as reported by the ISI web of Science because this currently the most authoritative source. In the future, other tools, based on Google Scholar (Harzing and van der Wai 2008) might be used to investigate to which extent the ISI web of science underestimates the scientific impact of papers on Neglected Tropical Zoonoses.

Third, the matched diseases employed in this paper contain some conditions that are not strictly considered as Neglected Tropical Disease but do mostly occur in the same populations as the Neglected Tropical Zoonoses For example, 75\% of the global burden of Vitamin A deficiency occurs in Africa. Removing such diseases from the list of employed diseases increases the gap between Tropical and non Tropical diseases. These three effects add to the discrepancy reported on in this paper.

\section{References}

Bakker, P., \& Rigter, H. (1985). Editors of medical journals: Who and from where. Scientometrics, 7(1), 11-22. Balasegaram, M., Balasegaram, S., Malvy, D., \& Millet, P. (2008). Neglected diseases in the news: A content analysis of recent international media coverage focussing on leishmaniasis and trypanosomiasis. PLoS Neglected Tropical Diseases, 2(5), e234.

Bosman, M., \& Mwinga, A. (2000). Tropical diseases and the 10/90 gap. The Lancet, 356(Supplement 1), S63-S63.

Catling, J., Mason, V., \& Upton, D. (2009). Quality is in the eye of the beholder? An evaluation of impact factors and perception of journal prestige in the UK. Scientometrics, 81(2), 333-345.

Chew, M., Villanueva, E., \& Van Der Weyden, M. (2007). Life and times of the impact factor: Retrospective analysis of trends for seven medical journals (1994-2005) and their Editors' views. JRSM, $100(3), 142$.

Chirac, P., \& Torreele, E. (2006). Global framework on essential health R\&D. The Lancet, 367(9522), $1560-1561$.

Engels, D., \& Savioli, L. (2006). Reconsidering the underestimated burden caused by neglected tropical diseases. Trends in Parasitology, 22(8), 363-366.

Falagas, M., Pitsouni, E., Malietzis, G., \& Pappas, G. (2008). Comparison of PubMed, Scopus, web of science, and Google scholar: Strengths and weaknesses. The FASEB Journal, 22(2), 338.

Frank, M. (2003). Impact factors: Arbiter of excellence? Journal of the Medical Library Association, 91(1), 4.

Gordon, M. (1984). How authors select journals: A test of the reward maximization model of submission behaviour. Social Studies of Science, 14(1), 27-43.

Harzing, A., \& van der Wai, R. (2008). Google scholar as a new source for citation analysis. Ethics in Science and Environmental Politics (ESEP), 8(1), 61-73.

Horton, R. (2003). Medical journals: Evidence of bias against the diseases of poverty. Lancet, 361(9359), 712.

Hotez, P., Molyneux, D., Fenwick, A., Kumaresan, J., Sachs, S., Sachs, J., \& Savioli, L. (2007a). Control of neglected tropical diseases. The New England Journal of medicine, 357(10), 1018.

Hotez, P., Molyneux, D., Fenwick, A., Ottesen, E., Sachs, S., \& Sachs, J. (2007b). Incorporating a rapidimpact package for neglected tropical diseases with programs for HIV/AIDS, tuberculosis, and malaria. PLoS Medicine, 3(5), 576.

Hotez, P., Fenwick, A., Savioli, L., \& Molyneux, D. (2009). Rescuing the bottom billion through control of neglected tropical diseases. The Lancet, 373(9674), 1570-1575.

Hunter, P. (2009). Bibliometrics, research quality, and neglected tropical diseases. The Lancet, 373(9664), $630-631$.

Krell, F. (2010). Should editors influence journal impact factors? Learned Publishing, 23(1), 59-62.

Luukkonen, T. (1992). Is scientists' publishing behaviour reward seeking? Scientometrics, 24(2), $297-319$.

Mathers, C., Ezzati, M., Lopez, A. (2007). Measuring the burden of neglected tropical diseases: The global burden of disease framework. PLoS Neglected Tropical Diseases, 1(2), e161.

Matias-Guiu, J., Garcia-Ramos, R. (2008). The impact factor and editorial decisions. Neurologia, 23(6), $342-348$. 
Médecins Sans Frontières (2001). Fatal imbalance: The crisis in research and development for drugs for neglected diseases. Tech. Rep. Geneva, Switzerland: MS Frontires.

Morel, C. M., Serruya, S. J., Penna, G. O., \& Guimares, R. (2009). Co-authorship network analysis: A powerful tool for strategic planning of research, development and capacity building programs on neglected diseases. PLoS Neglected Tropical Diseases, 3(8), e501.

Pringle, J. (2008). Trends in the use of ISI citation databases for evaluation. Learned Publishing, 21(2), 85-91.

Pruss-Ustun, A., Mathers, C., Corvalan, C., \& Woodward, A. (2003). The global burden of disease concept. Introduction and methods: Assessing the environmental burden of disease at national and local levels (pp. 27-40). Geneva, Switzerland: WHO.

R Development Core Team (2010). R: A language and environment for statistical computing. R Foundation for Statistical Computing, Vienna. http://www.R-project.org. Accessed Nov 2010.

Regazzi, J., \& Aytac, S. (2008). Author perceptions of journal quality. Learned Publishing, 21(3), 225-235.

Saha, S., Saint, S., \& Christakis, D. (2003). Impact factor: A valid measure of journal quality? JournalMedical Library Association, 91, 42-46.

Seglen, P. O. (1997). Why the impact factor of journals should not be used for evaluating research. British Medical Journal, 314(7079):497. http://www.bmj.com/content/314/7079/497.1.short.

Sumathipala, A., Siribaddana, S., \& Patel, V. (2004). Under-representation of developing countries in the research literature: Ethical issues arising from a survey of five leading medical journals. BMC Medical Ethics, 5(1), 5.

US National Library of Medicine (2010). Medline pubmed xml element descriptions and their attributes. Bethesda, Maryland, USA.

Vanderelst, D., \& Speybroeck, N. (2010). Quantifying the lack of scientific interest in neglected tropical diseases. PLoS Neglected Tropical Diseases, 4(1), 576.

Venables, W., \& Ripley, B. (2002). Modern applied statistics with S. New York, NY: Springer Verlag.

WHO (2008). The global burden of disease: 2004 Update. http://www.who.int/evidence/bod. Accessed Nov 2010.

WHO (2010). Working to overcome the global impact of neglected tropical diseases: First who report on neglected tropical diseases. Tech. Rep. Geneva, Switzerland: WHO.

WHO (2011). Neglected zoonotic diseases. http://www.who.int/neglected_diseases/zoonoses/en/. Accessed Nov 2010.

Yamey, G. (2002). The world's most neglected diseases Ignored by the pharmaceutical industry and by public-private partnerships. British Medical Journal, 325(7357), 176-177. 